Appl. Set-Valued Anal. Optim. 3 (2021), No. 3, pp. 281-292

Available online at http://asvao.biemdas.com

https://doi.org/10.23952/asvao.3.2021.3.03

\title{
ON CLARKE'S SUBDIFFERENTIAL OF MARGINAL FUNCTIONS
}

\author{
GEMAYQZEL BOUZA $^{1}$, ERNEST QUINTANA $^{2, *}$, CHRISTIANE TAMMER $^{3}$ \\ ${ }^{1}$ Faculty of Mathematics and Computer Science, University of Havana, Havana, Cuba \\ ${ }^{2}$ Institute for Mathematics, Technische Universität Ilmenau, Ilmenau, Germany \\ ${ }^{3}$ Institute of Mathematics, Martin-Luther-Universität Halle-Wittenberg, Halle (Saale), Germany
}

\begin{abstract}
In this paper, we derive an upper estimate of the Clarke subdifferential of marginal functions in Banach spaces. The structure of the upper estimate is very similar to other results already obtained in the literature. The novelty lies on the fact that we derive our assertions in general Banach spaces, and avoid the use of the Asplund assumption.
\end{abstract}

Keywords. Clarke's subdifferential; Coderivatives; Convexity; Marginal functions.

\section{INTRODUCTION}

Marginal functions are maps $\varphi: X \rightarrow \overline{\mathbb{R}}$ of the form

$$
\varphi(x):=\inf _{y \in F(x)} f(x, y),
$$

where $X$ and $Y$ are normed spaces, $\overline{\mathbb{R}}:=[-\infty,+\infty]$ is the extended set of real numbers, $F: X \rightrightarrows Y$ is a given set-valued mapping, and $f: X \times Y \rightarrow \overline{\mathbb{R}}$ is a given functional. Due to its numerous applications, the functionals of this type are among the most important ones in variational analysis, mathematical programming, and control theory. The study of subdifferentiability properties of $\varphi$, and specifically the computation of its subdifferential (in a specific sense), is therefore a relevant research topic.

Under convexity assumptions on the graph of $F$ and the functional $f$, the Fenchel subdifferential of $\varphi$ is analyzed in $[1,2,3,4]$. In this case, because of the nice convexity structure of the problem, the derived formulas are exact under mild conditions. On the other hand, the nonconvex setting has also been studied extensively; see, e.g., [5, 6, 7, 8, 9]. However, in this case, the exactness is already too difficult to ensure. These results are then focused on computing upper estimates of the different types of subdifferentials of the marginal function. In [5], Bot studied the Clarke subdifferential of $\varphi$ when both $X$ and $Y$ are finite dimensional, $F$ is constant, and $f$ is given as the pointwise maxima of a finite number of continuously differentiable functions. A similar study was considered by Hiriart-Urruty in [6] for the case in which $\varphi$ is locally Lipschitz (in particular, when $F$ is locally Lipschitz). Later, in [7, 8, 9], the upper estimates of Fréchet

${ }^{*}$ Corresponding author.

E-mail addresses: gema@matcom.uh.cu (G. Bouza), ernest.quintana-aparicio@tu-ilmenau.de (E. Quintana), christiane.tammer@mathematik.uni-halle.de (C. Tammer).

Received February 10, 2021; Accepted June 12, 2021.

(C)2021 Applied Set-Valued Analysis and Optimization 
and Mordukhovich's subdifferential of $\varphi$ were derived by assuming that $X$ and $Y$ are Asplund spaces and that $F$ has a closed graph.

To the best of our knowledge, upper estimates for Clarke's subdifferential of $\varphi$ have not been studied in the literature without the Asplund assumption on the underlying spaces. In this paper, we address this case. Our results rely only on the Banach structure of $X$ and $Y$, and the Aubin property of $F$ at notable points.

The rest of the paper is organized as follows. In Section 2, we establish important notations and definitions used throughout the text. In Section 3, the main results are derived. We conclude with some remarks and future lines of research in Section 4, the last section.

\section{PRELIMINARIES}

We start by establishing the main notations, which will be used later. Given a normed space $\left(X,\|\cdot\|_{X}\right)$, we denote by $\left(X^{*},\|\cdot\|_{X}^{*}\right)$ its topological dual. In addition, the closed unit balls in $X$ and $X^{*}$ are be denoted as $\mathbb{B}_{X}$ and $\mathbb{B}_{X}^{*}$, respectively. We omit the subscript $X$ if there is no risk of confusion. For a nonempty set $A \subseteq X$, int $A, \operatorname{cl} A, \operatorname{bd} A$, and conv $A$ stand for the interior, closure, boundary, and convex hull of $A$, respectively. Furthermore, if $B \subseteq X^{*}$, we denote the closure of the convex hull of $B$ in the weak topology of $X^{*}$ by $\overline{\operatorname{conv}}^{*} B$. Throughout the rest of this section, we assume that normed spaces $X$ and $Y$ are given.

Definition 2.1. Let $F: X \rightrightarrows Y$ be a set-valued mapping.

(i) The graph of $F$ is the set defined as

$$
\operatorname{gph} F:=\{(x, y) \in X \times Y \mid y \in F(x)\} .
$$

(ii) The domain of $F$ is the set given by

$$
\operatorname{dom} F:=\{x \in X \mid F(x) \neq \emptyset\} .
$$

(iii) We say that $F$ satisfies the Aubin property at $(\bar{x}, \bar{y}) \in \operatorname{gph} F$ if there exists $\ell \geq 0$, together with neighborhoods $U$ of $\bar{x}$ and $V$ of $\bar{y}$, such that

$$
\forall x, x^{\prime} \in U: \quad F(x) \cap V \subseteq F\left(x^{\prime}\right)+\ell\left\|x-x^{\prime}\right\|_{X} \mathbb{B}_{Y}
$$

Definition 2.2. Let $f: X \rightarrow \overline{\mathbb{R}}$ be an extended real valued functional.

(i) The domain of $f$ is the set

$$
\operatorname{dom} f:=\{x \in X \mid f(x)<+\infty\} .
$$

(ii) The epigraph of $f$ is the set defined as

$$
\text { epi } f:=\{(x, t) \in X \times \mathbb{R} \mid f(x) \leq t\} .
$$

(iii) We say that $f$ is convex if epi $f$ is a convex set.

(iv) We say that $f$ is Lipschitz on a set $A \subseteq X$ provided that $f$ is finite on $A$ and there exists $L>0$ such that

$$
\forall x, x^{\prime} \in A:\left|f(x)-f\left(x^{\prime}\right)\right| \leq L\left\|x-x^{\prime}\right\|_{X}
$$


We say that $f$ is locally Lipschitz at $\bar{x}$ if there is a neighborhood $U$ of $\bar{x}$ such that $f$ is Lipschitz on $U$. In addition, $f$ is said to be locally Lipschitz on $A$ if $f$ is locally Lipschitz at every point $x \in A$. Thus, in particular, $A \subseteq \operatorname{int} \operatorname{dom} f$.

An important class of extended real valued functionals that will be useful in the sequel are the so called support functions of subsets in a dual space. Formally, for a set $A \subseteq X^{*}$, this is the functional $\sigma_{A}: X \rightarrow \overline{\mathbb{R}}$ defined as

$$
\sigma_{A}(x):=\sup _{x^{*} \in A}\left\langle x^{*}, x\right\rangle .
$$

Next, we present the main concepts of the generalized differentiation that will be employed in the paper. The material in this part is mostly classical, and we refer the reader to [10] for a more in depth discussion of these concepts and their properties.

Definition 2.3. Let $f: X \rightarrow \overline{\mathbb{R}}$ be locally Lipschitz at a given point $\bar{x} \in \operatorname{dom} f$.

(i) For each $u \in X$, the generalized directional derivative of $f$ at $\bar{x}$ in the direction $u$ is defined by

$$
f^{\circ}(\bar{x}, u):=\limsup _{x \rightarrow \bar{x}, t \downarrow 0} \frac{f(x+t u)-f(x)}{t} .
$$

(ii) The Clarke subdifferential of $f$ at $\bar{x} \in \operatorname{dom} f$ is the set defined as

$$
\partial^{\circ} f(\bar{x}):=\left\{x^{*} \in X^{*} \mid \forall u \in X: f^{\circ}(\bar{x}, u) \geq\left\langle x^{*}, u\right\rangle\right\} .
$$

Clarke's subdifferential generalizes the well known concept of the Fenchel subdifferential of a convex functional to the locally Lipschitz setting; see Proposition 2.1 (v) below. For a convex functional $f$ and the point $\bar{x} \in \operatorname{dom} f$, this is the set

$$
\partial f(\bar{x}):=\left\{x^{*} \in X^{*} \mid \forall x \in X:\left\langle x^{*}, x-\bar{x}\right\rangle \leq f(x)-f(\bar{x})\right\} .
$$

We continue by defining the Clarke's tangent and normal cones to a set.

Definition 2.4. Let $A \subseteq X$ be nonempty and let $\bar{x} \in A$.

(i) The Clarke tangent cone to $A$ at $\bar{x}$ is the set defined as

$$
T(A, \bar{x}):=\left\{u \in X \mid \begin{array}{l}
\forall\left\{x_{k}\right\}_{k \geq 1} \subseteq A,\left\{t_{k}\right\}_{k \geq 1} \subseteq \mathbb{R} \text { with } x_{k} \rightarrow \bar{x}, t_{k} \downarrow 0: \\
\exists\left\{u_{k}\right\}_{k \geq 1} \text { such that } u_{k} \rightarrow u \text { and } x_{k}+t_{k} u_{k} \in A \forall k
\end{array}\right\} .
$$

A vector $u \in T(A, \bar{x})$ is called a tangent to $A$ at $\bar{x}$.

(ii) The Clarke normal cone of $A$ at $\bar{x}$ is the set defined by

$$
N_{C}(A, \bar{x}):=\left\{x^{*} \in X^{*} \mid \forall u \in T(A, \bar{x}):\left\langle x^{*}, u\right\rangle \leq 0\right\} .
$$

We can now define the coderivative of a set-valued mapping in the sense of Clark.

Definition 2.5. Let $F: X \rightrightarrows Y$ be a given set-valued mapping and let $(\bar{x}, \bar{y}) \in \operatorname{gph} F$. The Clarke coderivative of $F$ at $(\bar{x}, \bar{y})$ is the set-valued mapping $D_{C}^{*} F(\bar{x}, \bar{y}): Y^{*} \rightrightarrows X^{*}$ defined as

$$
D_{C}^{*} F(\bar{x}, \bar{y})\left(y^{*}\right):=\left\{x^{*} \in X^{*} \mid\left(x^{*},-y^{*}\right) \in N_{C}(\operatorname{gph} F,(\bar{x}, \bar{y}))\right\} .
$$

We close this section with the following proposition that collects some properties of the Clarke's subdifferential that are used in the main results; see [10] for the proofs. 
Proposition 2.1. Let $X$ be a Banach space and $f, g: X \rightarrow \overline{\mathbb{R}}$ be locally Lipschitz at $\bar{x} \in X$. The following statements hold.

(i) The set $\partial^{\circ} f(\bar{x})$ is nonempty, convex, and $w^{*}$ - compact (that is, compact w.r.t. the $w^{*}$ topology).

(ii) $\partial^{\circ}(f+g)(\bar{x}) \subseteq \partial^{\circ} f(\bar{x})+\partial^{\circ} g(\bar{x})$. Equality holds if $f$ and $g$ are convex.

(iii) $f^{\circ}(\bar{x}, \cdot)=\sigma_{\partial \circ f(\bar{x})}(\cdot)$.

(iv) If $\bar{x}$ is a local minimum of $f$, then $0 \in \partial^{\circ} f(\bar{x})$.

(v) If $f$ is convex, then $\partial^{\circ} f(\bar{x})=\partial f(\bar{x})$.

\section{MAin Results}

The basic setting in which we derive the results of this section is the following.

Assumption 3.1. Let $X$ and $Y$ be Banach spaces, and let $F: X \rightrightarrows Y$ be a set-valued mapping with $F(x) \neq \emptyset$ for every $x \in X$. Furthermore, let $f: X \times Y \rightarrow \overline{\mathbb{R}}$ be a given extended real valued functional, and consider the associated marginal function $\varphi: X \rightarrow \overline{\mathbb{R}}$ given in (1.1). In addition, consider the set-valued solution mapping $S: X \rightrightarrows Y$ defined as

$$
S(x):=\{y \in F(x): f(x, y)=\varphi(x)\} .
$$

We start by proving some lemmata that contribute to the main theorem. The assertion in the next lemma is proved by using Ekeland's variational principle; see e.g., [11, 12, 13].

Lemma 3.1. Let $g: Y \rightarrow \mathbb{R}$ be a given functional, and suppose that $g$ is convex, continuous, and bounded from below. Then, there are sequences $\left\{v_{k}\right\}_{k \geq 1} \subseteq Y$ and $\left\{y_{k}^{*}\right\}_{k \geq 1} \subseteq Y^{*}$ such that:

(i) $g\left(v_{k}\right) \rightarrow \inf _{y \in Y} g(y)$,

(ii) $y_{k}^{*} \in \partial g\left(v_{k}\right)$,

(iii) $\left|\left\langle y_{k}^{*}, v_{k}\right\rangle\right| \rightarrow 0$,

(iv) $y_{k}^{*} \rightarrow 0$.

Proof. Let $\left\{y_{k}\right\}_{k \geq 1}$ be a minimizing sequence such that

$$
g\left(y_{k}\right)<\inf _{y \in Y} g(y)+\frac{1}{k} .
$$

By Ekeland's variational principle [13, Theorem 8.2.4], for every $\lambda_{k}>0$, we can find $v_{k} \in Y$ such that

(a) $\left\|y_{k}-v_{k}\right\| \leq \lambda_{k}$

(b) $g\left(v_{k}\right) \leq \inf _{y \in Y} g(y)+\frac{2}{k}$, and

(c) $v_{k}$ is a minimizer of the functional

$$
g_{k}(\cdot):=g(\cdot)+\frac{1}{k \lambda_{k}}\left\|\cdot-v_{k}\right\| .
$$

Since $g$ is a continuous convex functional, it follows that $g_{k}$ is also convex and continuous. Hence, from (c) and items (iv), (v), and (ii) in Proposition 2.1, we deduce that

$$
0 \in \partial g_{k}\left(v_{k}\right)=\partial g\left(v_{k}\right)+\frac{1}{k \lambda_{k}} \mathbb{B}^{*} .
$$


From (3.1), we then obtain the existence of $y_{k}^{*} \in \partial g\left(v_{k}\right)$ with

$$
\left\|y_{k}^{*}\right\|_{*} \leq \frac{1}{k \lambda_{k}}
$$

On the other hand, we have from (a) that

$$
\left\|v_{k}\right\| \leq\left\|y_{k}\right\|+\lambda_{k}
$$

The inequalities (3.2) and (3.3) now imply

$$
\left|\left\langle y_{k}^{*}, v_{k}\right\rangle\right| \leq\left\|y_{k}^{*}\right\|_{*}\left\|v_{k}\right\| \leq \frac{\lambda_{k}+\left\|y_{k}\right\|}{k \lambda_{k}} .
$$

Since $v_{k}$ and $y_{k}^{*}$ depend on $\lambda_{k}$, we next show that $\lambda_{k}$ can be chosen such that the properties $(i)-(i v)$ in the statement are true. Indeed, independently of the choice of the sequence $\left\{\lambda_{k}\right\}_{k \geq 1}$, we obtain properties $(i)$ and (ii) from (b) and (3.1), respectively. In order to guarantee (iii) and (iv), we set $\lambda_{k}:=\left\|y_{k}\right\|+1$ for every $k \in \mathbb{N}$. Then, from (3.2), we find that

$$
\left\|y_{k}^{*}\right\|_{*} \leq \frac{1}{k\left(\left\|y_{k}\right\|+1\right)} \leq \frac{1}{k} \rightarrow 0
$$

which proves (iii). Furthermore, it follows from (3.4) that

$$
\left|\left\langle y_{k}^{*}, v_{k}\right\rangle\right| \leq \frac{\lambda_{k}+\left\|y_{k}\right\|}{k \lambda_{k}}=\frac{2\left\|y_{k}\right\|+1}{k\left(\left\|y_{k}\right\|+1\right)} \leq \frac{2}{k} \rightarrow 0,
$$

and thus $(i v)$ is fulfilled. The proof is complete.

Before stating the next lemma, we give some notations. For a set $G \subseteq X^{*} \times Y^{*}$, the projection of $G$ onto $X^{*}$ is defined as

$$
G_{X^{*}}:=\left\{x^{*} \in X^{*} \mid \exists y^{*} \in Y^{*}:\left(x^{*}, y^{*}\right) \in G\right\} .
$$

The projection of $G$ onto $Y^{*}$, denoted by $G_{Y^{*}}$, is defined similarly. For $(\bar{u}, \bar{v}) \in X \times Y$, we put

$$
G^{(\bar{u}, \bar{v})}:=\left\{\left(x^{*}, y^{*}\right) \in G \mid\left\langle\left(x^{*}, y^{*}\right),(\bar{u}, \bar{v})\right\rangle=\sigma_{G}((\bar{u}, \bar{v}))\right\},
$$

and hence,

$$
G_{Y^{*}}^{(\bar{u}, \bar{v})}=\left\{y^{*} \in Y^{*} \mid \exists x^{*} \in X^{*} \text { such that }\left(x^{*}, y^{*}\right) \in G^{(\bar{u}, \bar{v})}\right\} .
$$

It is easy to see that the sets $G^{(\bar{u}, \bar{v})}$ and $G_{Y^{*}}^{(\bar{u}, \bar{v})}$ are convex and $w^{*}$ - closed if $G$ is convex and $w^{*}$ closed.

Lemma 3.2. Let $G$ be a $w^{*}$ - compact convex subset of $X^{*} \times Y^{*}$. Fix $\bar{u} \in X$ and consider the functional $g: Y \rightarrow \mathbb{R}$ defined as follows:

$$
g(v):=\sigma_{G}(\bar{u}, v) .
$$

Then, $g$ is a continuous convex functional satisfying

$$
\forall \bar{v} \in Y: \partial g(\bar{v})=G_{Y^{*}}^{(\bar{u}, \bar{v})} .
$$

Proof. The statement will be a consequence of [13, Proposition 4.5.2]. Indeed, we see that $G$ with the $w^{*}$ - topology is a compact Hausdorff space. Now, consider, for $\left(x^{*}, y^{*}\right) \in G$, the functional $g_{\left(x^{*}, y^{*}\right)}: Y \rightarrow \mathbb{R}$ defined by $g_{\left(x^{*}, y^{*}\right)}(v):=\left\langle y^{*}, v\right\rangle+\left\langle x^{*}, \bar{u}\right\rangle$. Obviously, every functional of this type is convex and continuous at $\bar{v}$. Furthermore, we have $g(v)=\sup _{\left(x^{*}, y^{*}\right) \in G} g_{\left(x^{*}, y^{*}\right)}(v)$ 
for every $v \in Y$. Moreover, because of the definition of the $w^{*}$-topology, the map $\left(x^{*}, y^{*}\right) \rightarrow$ $g_{\left(x^{*}, y^{*}\right)}(v)$ is continuous (and hence upper semicontinuous) for any $v \in Y$.

On the other hand, note that $G^{(\bar{u}, \bar{v})}=\left\{\left(x^{*}, y^{*}\right) \in G \mid g_{\left(x^{*}, y^{*}\right)}(\bar{v})=g(\bar{v})\right\}$ and that $\partial g_{\left(x^{*}, y^{*}\right)}(\bar{v})=$ $\left\{y^{*}\right\}$. Taking this into account and applying [13, Proposition 4.5.2], we obtain

$$
\begin{aligned}
\partial g(\bar{v}) & =\overline{\operatorname{conv}}^{*}\left(\bigcup_{\left(x^{*}, y^{*}\right) \in G^{(\bar{u}, \bar{v})}} \partial g_{\left(x^{*}, y^{*}\right)}(\bar{v})\right) \\
& =\overline{\operatorname{conv}}^{*}\left(\bigcup_{\left(x^{*}, y^{*}\right) \in G^{(\bar{u}, \bar{v})}}\left\{y^{*}\right\}\right) \\
& =\overline{\operatorname{conv}}^{*}\left(G_{Y^{*}}^{(\bar{u}, \bar{v})}\right) .
\end{aligned}
$$

Thus, the statement follows from the fact that $G_{Y^{*}}^{(\bar{u}, \bar{v})}$ is $w^{*}$ - closed and convex.

To proceed, we need the notions of inner semicompactness and closedness of a set-valued mapping. The inner semicompactness and closedness of the solution mapping $S$ from Assumption 3.1 are standard hypotheses when deriving estimates for subdifferentials of marginal functions; see, e.g., [7, Theorem 1.108 and Theorem 3.38].

Definition 3.1. Let $\bar{x} \in \operatorname{dom} S$. We say that:

(i) $S$ is inner semicompact at $\bar{x}$ if $\bar{x} \in$ int $(\operatorname{dom} S)$, and, for every sequence $x_{k} \rightarrow \bar{x}$, there is a sequence $y_{k} \in S\left(x_{k}\right)$ that contains a convergent subsequence as $k \rightarrow \infty$;

(ii) $S$ is closed at $\bar{x}$ if, for any sequence $\left\{\left(x_{k}, y_{k}\right)\right\}_{k \geq 1} \subseteq \operatorname{gph} S$ with $\left(x_{k}, y_{k}\right) \rightarrow(\bar{x}, \bar{y}),(\bar{x}, \bar{y}) \in$ gph $S$.

Remark 3.1. By Weierstrass's Theorem [14, Theorem 2.3], it is straightforward to verify that, in our context, the set-valued mapping $S$ is inner semicompact at $\bar{x}$ if the following assumptions are fulfilled:

(i) $Y$ is finite dimensional;

(ii) there exists a neighborhood $U$ of $\bar{x}$ such that

- $f$ is lower semicontinuous (l.s.c) on $U$;

- $F(x)$ is compact for every $x \in U$;

- the set $F[U]$ is bounded.

Remark 3.2. A sufficient condition for the closedness of $S$ at $\bar{x}$ is that

(i) $\varphi$ is continuous at $\bar{x}$;

(ii) $f$ is l.s.c on $\{\bar{x}\} \times F(\bar{x})$;

(iii) $F$ is closed at $\bar{x}$.

Indeed, let $\left\{\left(x_{k}, y_{k}\right)\right\}_{k \geq 1} \subseteq$ gph $S$ be convergent to $(\bar{x}, \bar{y})$. Then, for every $k \in \mathbb{N}$, we have $f\left(x_{k}, y_{k}\right)=\varphi\left(x_{k}\right)$. Because $\varphi$ is continuous at $\bar{x}$, it follows that $f\left(x_{k}, y_{k}\right) \rightarrow \varphi(\bar{x})$. Since $S\left(x_{k}\right) \subseteq$ $F\left(x_{k}\right)$ for every $k \in \mathbb{N}$, and $F$ is closed at $\bar{x}$, we obtain $\bar{y} \in F(\bar{x})$. Hence, we also have that $f$ is 1.s.c at $(\bar{x}, \bar{y})$. From this, we have

$$
f(\bar{x}, \bar{y}) \leq \liminf _{k \rightarrow \infty} f\left(x_{k}, y_{k}\right)=\varphi(\bar{x})
$$


which implies $\bar{y} \in S(\bar{x})$.

In the following lemma, we analyze the Clarke's subdifferential of the marginal function $\varphi$ in the unconstrained case $F(\cdot):=Y$. This is a weaker version of our main result that will be derived in Theorem 3.1.

Lemma 3.3. Let $\bar{x} \in X$ and suppose that

(i) $\forall x \in X: F(x):=Y$;

(ii) $f$ is locally Lipschitz on $\{\bar{x}\} \times S(\bar{x})$;

(iii) the associated marginal function $\varphi$ is locally Lipschitz at $\bar{x} \in X$;

(iv) the set-valued solution mapping $S$ is inner semicompact and closed at $\bar{x}$.

Then,

$$
\partial^{\circ} \varphi(\bar{x}) \subseteq \overline{\operatorname{conv}}^{*}\left(\bigcup_{\bar{y} \in S(\bar{x})}\left\{x^{*} \in X^{*}:\left(x^{*}, 0\right) \in \partial^{\circ} f(\bar{x}, \bar{y})\right\}\right) .
$$

Proof. Consider the set-valued mapping $\tilde{S}: X \rightrightarrows Y$ defined as

$$
\tilde{S}(x):=\left\{y \in S(x): \partial^{\circ} f(x, y) \cap\left(X^{*} \times\{0\}\right) \neq \emptyset\right\} .
$$

Then, it suffices to take the inclusion (3.6) over $\bar{y} \in \tilde{S}(\bar{x})$ in the proof. We need the following claims.

- Claim 1: The inequality below holds:

$$
\varphi^{\circ}(\bar{x}, \cdot) \leq \sup _{\bar{y} \in S(\bar{x})} \inf _{v \in Y} f^{\circ}((\bar{x}, \bar{y}),(\cdot, v)) .
$$

Indeed, fix any $u \in X$. Then, we can find sequences $\left\{x_{k}\right\}_{k \geq 1} \subseteq X$ and $\left\{t_{k}\right\}_{k \geq 1} \downarrow 0$ such that $x_{k} \rightarrow \bar{x}$ and

$$
\varphi^{\circ}(\bar{x}, u)=\lim _{k \rightarrow \infty} \frac{\varphi\left(x_{k}+t_{k} u\right)-\varphi\left(x_{k}\right)}{t_{k}} .
$$

Since $S$ is inner semicompact at $\bar{x}$, there exists a sequence $\left\{y_{k}\right\}_{k \geq 1} \subseteq Y$ such that $y_{k} \in$ $S\left(x_{k}\right)$ for every $k \in \mathbb{N}$, and $\left\{y_{k}\right\}_{k \geq 1}$ contains a convergent subsequence. Without loss of generality, we assume that $y_{k} \rightarrow \bar{y}$ for some $\bar{y} \in Y$. Since $S$ is closed at $\bar{x}$, it follows that $\bar{y} \in S(\bar{x})$. Then, taking into account (3.7), and the definition of $\varphi$, for any $v \in Y$ we have

$$
\begin{aligned}
\varphi^{\circ}(\bar{x}, u) & \leq \limsup _{k \rightarrow \infty} \frac{f\left(x_{k}+t_{k} u, y_{k}+t_{k} v\right)-f\left(x_{k}, y_{k}\right)}{t_{k}} \\
& \leq \limsup _{(x, y) \rightarrow(\bar{x}, \bar{y}), t \downarrow 0} \frac{f(x+t u, y+t v)-f(x, y)}{t} \\
& =f^{\circ}((\bar{x}, \bar{y}),(u, v)) .
\end{aligned}
$$

Taking now the infimum over $v \in Y$ in the above inequality, we obtain the desired result. - Claim 2: $\tilde{S}(\bar{x}) \neq \emptyset$.

We show that the element $\bar{y} \in S(\bar{x})$ constructed in the proof of Claim 1 satisfies $\bar{y} \in$ $\tilde{S}(\bar{x})$. Indeed, fix any $\bar{u} \in X$. Then, from Claim 1 , we have

$$
-\infty<\varphi^{\circ}(\bar{x}, \bar{u}) \leq \inf _{v \in Y} f^{\circ}((\bar{x}, \bar{y}),(\bar{u}, v)) .
$$


Set $G:=\partial^{\circ} f(\bar{x}, \bar{y})$ and consider the sets $G_{X^{*}}, G_{Y^{*}}$. Then, by Proposition 2.1 (i), we know that $G$ is nonempty, convex, and $w^{*}$ - compact. It is then straightforward to verify that $G_{X^{*}}$ and $G_{Y^{*}}$ are also nonempty, convex, and $w^{*}$ - compact sets. Taking into account now (3.8) and Proposition 2.1 (iii), we deduce that

$$
-\infty<\inf _{v \in Y} \sigma_{G}(\bar{u}, v) \leq \sigma_{G_{X^{*}}}(\bar{u})+\inf _{v \in Y} \sigma_{G_{Y^{*}}}(v) .
$$

Thus, in particular,

$$
\inf _{v \in Y} \sigma_{G_{Y^{*}}}(v)>-\infty .
$$

We now show that $0 \in G_{Y^{*}}$. Indeed, otherwise we could strongly separate the sets $\{0\}$ and $G_{Y^{*}}$ (see, e.g., [15, Theorem 2.2.8.]) to obtain a vector $\bar{v} \in Y$ such that

$$
0=\langle 0, \bar{v}\rangle>\sigma_{G_{Y^{*}}}(\bar{v}) .
$$

Hence, taking into account the positive homogeneity of $\sigma_{G_{Y^{*}}}$, we obtain

$$
-\infty=\lim _{\lambda \rightarrow \infty} \sigma_{G_{Y^{*}}}(\lambda \bar{v})
$$

which is a contradiction to (3.9).

By the definition of $G_{Y^{*}}$, we conclude that there exists $x^{*} \in X^{*}$ such that $\left(x^{*}, 0\right) \in G$, or equivalently, $\bar{y} \in \tilde{S}(\bar{x})$.

- Claim 3: For $\bar{y} \in \tilde{S}(\bar{x})$ and $\bar{u} \in X$, we have

$$
\sup _{\left(x^{*}, 0\right) \in \partial^{\circ} f(\bar{x}, \bar{y})}\left\langle x^{*}, \bar{u}\right\rangle=\inf _{v \in Y} f^{\circ}((\bar{x}, \bar{y}),(\bar{u}, v)) .
$$

In order to prove (3.10), let us use the notation of Claim 2 and the function $g$ defined by (3.5). By Lemma 3.2, we have

$$
\forall v \in Y: \partial g(v)=G_{Y^{*}}^{(\bar{u}, v)} .
$$

Furthermore, because $g$ is continuous, convex and bounded from below, it is possible to apply Lemma 3.1. Therefore, we obtain the existence of sequences $\left\{v_{k}\right\} \subseteq Y$ and $\left\{y_{k}^{*}\right\}_{k \geq 1} \subseteq Y^{*}$ such that, for every $k \in \mathbb{N}$,

(a) $g\left(v_{k}\right) \rightarrow \inf _{y \in Y} g(y)$;

(b) $y_{k}^{*} \in G_{Y^{*}}^{\left(\bar{u}, v_{k}\right)}$;

(c) $\left|\left\langle y_{k}^{*}, v_{k}\right\rangle\right| \rightarrow 0$;

(d) $y_{k}^{*} \rightarrow 0$.

In particular, from (b), we conclude the existence of a sequence $\left\{x_{k}^{*}\right\}_{k \geq 1} \subseteq G_{X^{*}}$ such that, for every $k \in \mathbb{N}$, the inclusion $\left(x_{k}^{*}, y_{k}^{*}\right) \in G$ holds, and

$$
g\left(v_{k}\right)=\left\langle x_{k}^{*}, \bar{u}\right\rangle+\left\langle y_{k}^{*}, v_{k}\right\rangle .
$$

Since $G$ is $w^{*}$ - compact, the net $\left\{\left(x_{k}^{*}, y_{k}^{*}\right)\right\}_{k \geq 1}$ has a convergent subnet. Without loss of generality, let $\left(x_{k}^{*}, y_{k}^{*}\right) \stackrel{w^{*}}{\rightarrow}\left(\bar{x}^{*}, \bar{y}^{*}\right) \in G$. By (d), we get in particular $\bar{y}^{*}=0$ and $\left(\bar{x}^{*}, 0\right) \in G$. Taking into account (a) and (c), and taking the limit in (3.11), we obtain

$$
\left\langle\bar{x}^{*}, \bar{u}\right\rangle=\inf _{v \in Y} g(v) .
$$


This means that

$$
\begin{array}{ccl}
\sup _{\left(x^{*}, 0\right) \in \partial^{\circ} f(\bar{x}, \bar{y})}\left\langle x^{*}, \bar{u}\right\rangle \geq\left\langle\bar{x}^{*}, \bar{u}\right\rangle & \stackrel{(3.12)}{=} & \inf _{v \in Y} g(v) \\
& \stackrel{(3.5)}{=} & \inf _{v \in Y} \sigma_{G}(\bar{u}, v) \\
& \text { (Proposition 2.1 (iii)) } & \inf _{v \in Y} f^{\circ}((\bar{x}, \bar{y}),(\bar{u}, v)) .
\end{array}
$$

In order to see the validity of the reverse inequality note that if $\left(x^{*}, 0\right) \in G$, then we obtain from the definition of $\sigma_{G}$ that

$$
\forall(u, v) \in X \times Y:\left\langle x^{*}, u\right\rangle \leq \sigma_{G}(u, v) .
$$

Fixing $u=\bar{u}$ and taking the infimum over $v \in Y$, the desired inequality is obtained.

Now, assume that

$$
\bar{x}^{*} \notin \overline{\operatorname{conv}}^{*}\left(\bigcup_{\bar{y} \in \tilde{S}(\bar{x})}\left\{x^{*} \in X^{*}:\left(x^{*}, 0\right) \in \partial^{\circ} f(\bar{x}, \bar{y})\right\}\right) .
$$

By the classical strong separation theorem (see, e.g., [15, Theorem 2.2.8.]), we then find $\bar{u} \in X$ such that

$$
\left\langle\bar{x}^{*}, \bar{u}\right\rangle>\sup _{\bar{y} \in \tilde{S}(\bar{x})\left(x^{*}, 0\right) \in \partial^{\circ} f(\bar{x}, \bar{y})}\left\langle x^{*}, \bar{u}\right\rangle .
$$

However, because of Claim 3, we know that (3.10) holds. Putting this back into (3.13), and using Claim 1, we obtain

$$
\left\langle\bar{x}^{*}, \bar{u}\right\rangle>\sup _{\bar{y} \in \tilde{S}(\bar{x})} \inf _{v \in Y} f^{\circ}((\bar{x}, \bar{y}),(\bar{u}, v)) \geq \varphi^{\circ}(\bar{x}, \bar{u}) .
$$

This means that $\bar{x}^{*} \notin \partial^{\circ} \varphi(\bar{x})$. Hence, the desired inclusion holds. The proof is complete.

Remark 3.3. Suppose that $X=\mathbb{R}^{n}, Y=\mathbb{R}^{m}$, and that

$$
f(x, y)=\max \left\{f_{i}(x, y) \mid i=1, \ldots, s\right\}
$$

for some continuously differentiable functions $f_{i}: \mathbb{R}^{n} \times \mathbb{R}^{m} \rightarrow \mathbb{R}, i=1, \ldots, s$, and some $s \in \mathbb{N}$. Furthermore, consider the set-valued mapping $I: \mathbb{R}^{n} \times \mathbb{R}^{m} \rightrightarrows\{1, \ldots, s\}$ given by $I(x, y):=\{i \in$ $\left.\{1, \ldots, s\}: f_{i}(x, y)=f(x, y)\right\}$. Then, the upper estimate of $\partial^{\circ} \varphi(\bar{x})$ given in (3.6) is the closure of the one provided in [5, Theorem 4], where it was shown under different assumptions that

$$
\begin{aligned}
\partial^{\circ} \varphi(\bar{x}) \subseteq \operatorname{conv}( & \bigcup_{\bar{y} \in S(\bar{x})}\left\{\sum_{i \in I(\bar{x}, \bar{y})} \mu_{i} \nabla_{x} f_{i}(\bar{x}, \bar{y}): \mu_{i} \geq 0, \forall i \in I(\bar{x}, \bar{y}),\right. \\
& \left.\left.\sum_{i \in I(\bar{x}, \bar{y})} \mu_{i}=1, \sum_{i \in I(\bar{x}, \bar{y})} \mu_{i} \nabla_{y} f_{i}(\bar{x}, \bar{y})=0\right\}\right) .
\end{aligned}
$$

Indeed, because of the particular structure of $f$, in this case, we can apply [10, Proposition 2.3.12] to obtain

$$
\forall(x, y) \in \mathbb{R}^{n} \times \mathbb{R}^{m}: \partial^{\circ} f(x, y)=\operatorname{conv}\left\{\nabla f_{i}(x, y): i \in I(x, y)\right\} .
$$


Thus, in (3.6), we have that $\left(x^{*}, 0\right) \in \partial^{\circ} f(\bar{x}, \bar{y})$ if an only if, for each $i \in I(\bar{x}, \bar{y})$, there exists $\mu_{i} \geq 0$ such that $\sum_{i \in I(\bar{x}, \bar{y})} \mu_{i}=1, \sum_{i \in I(\bar{x}, \bar{y})} \mu_{i} \nabla_{y} f_{i}(\bar{x}, \bar{y})=0$, and $x^{*}=\sum_{i \in I(\bar{x}, \bar{y})} \mu_{i} \nabla_{x} f_{i}(\bar{x}, \bar{y})$. This implies the statement.

We can now state the main result of this paper.

Theorem 3.1. Let $\bar{x} \in X$ and suppose that

(i) F satisfies the Aubin property at every point of the set $\{\bar{x}\} \times S(\bar{x})$;

(ii) there is a neighborhood $U$ of $\bar{x}$ and a constant $L_{1}>0$ such that, for every $x \in U$,

- $f(x, \cdot)$ is Lipschitz on $Y$ with constant $L_{1}$;

- $F(x)$ is closed.

(iii) $f$ is locally Lipschitz on $\{\bar{x}\} \times S(\bar{x})$;

(iv) the associated marginal function $\varphi$ is Lipschitz around $\bar{x} \in X$;

(v) the set-valued solution mapping $S$ is inner semicompact and closed at $\bar{x}$.

Then,

$$
\partial^{\circ} \varphi(\bar{x}) \subseteq \overline{\operatorname{conv}}^{*}\left(\bigcup_{\substack{\bar{y} \in S(\bar{x}) \\\left(x^{*}, y^{*}\right) \in \partial^{\circ} f(\bar{x}, \bar{y})}}\left[x^{*}+D_{C}^{*} F(\bar{x}, \bar{y})\left(y^{*}\right)\right]\right) .
$$

Proof. Since $S$ is inner semicompact at $\bar{x}$, in particular, we have $\bar{x} \in \operatorname{int}(\operatorname{dom} S)$. Hence, without loss of generality, we can assume that $U \subseteq$ int $(\operatorname{dom} S)$. It follows that the assumptions of [10, Proposition 2.4.3] are fulfilled. Then, for any $L^{\prime}>L_{1}$ and every $x \in U$,

$$
\varphi(x)=\inf _{y \in Y}\left\{f(x, y)+L^{\prime} \Delta_{F}(x, y)\right\}
$$

and

$$
S(x)=\left\{y \in Y \mid f(x, y)+L^{\prime} \Delta_{F}(x, y)=\varphi(x)\right\},
$$

where the function $\Delta_{F}: X \times Y \rightarrow \mathbb{R}$ is given by $\Delta_{F}(x, y):=\inf _{u \in F(x)}\|y-u\|$. We consider $f_{1}$ : $X \times Y \rightarrow \mathbb{R}$ defined as

$$
f_{1}(x, y):=f(x, y)+L^{\prime} \Delta_{F}(x, y) .
$$

Therefore,

$$
\forall x \in U: \varphi(x)=\inf _{y \in Y} f_{1}(x, y) .
$$

Since $F$ satisfies the Aubin property at every point of the set $\{\bar{x}\} \times S(\bar{x})$, according to [7, Theorem 1.41], we have that $\Delta_{F}$ is locally Lipschitz at any point $(\bar{x}, \bar{y}) \in\{\bar{x}\} \times S(\bar{x})$. This, together with assumption (iii), implies that $f_{1}$ is also locally Lipschitz at every point of the set $\{\bar{x}\} \times S(\bar{x})$. Applying now Lemma 3.3, we obtain

$$
\partial^{\circ} \varphi(\bar{x}) \subseteq \overline{\operatorname{conv}}^{*}\left(\bigcup_{\bar{y} \in S(\bar{x})}\left\{x^{*} \in X^{*} \mid\left(x^{*}, 0\right) \in \partial^{\circ} f_{1}(\bar{x}, \bar{y})\right\}\right)
$$


From Proposition 2.1 (ii) and [9, Corollary 1.4 (ii)], we have

$$
\partial^{\circ} f_{1}(\bar{x}, \bar{y}) \subseteq \partial^{\circ} f(\bar{x}, \bar{y})+\partial^{\circ} \Delta_{F}(\bar{x}, \bar{y}) \subseteq \partial^{\circ} f(\bar{x}, \bar{y})+N_{C}(\operatorname{gph} F,(\bar{x}, \bar{y})) .
$$

Taking into account this with (3.15), we have

$$
\partial^{\circ} \varphi(\bar{x}) \subseteq \overline{\operatorname{conv}}^{*}\left(\bigcup_{\bar{y} \in S(\bar{x})}\left\{x^{*} \in X^{*} \mid\left(x^{*}, 0\right) \in \partial^{\circ} f(\bar{x}, \bar{y})+N_{C}(\operatorname{gph} F,(\bar{x}, \bar{y}))\right\}\right),
$$

which is equivalent to our statement.

Remark 3.4. If the spaces $X$ and $Y$ are Asplund, then an upper estimate of Clarke's subdifferential of $\varphi$ can be obtained by means of the result in [8, Theorem 7] (see also [9]) and the fact that $\partial^{\circ} \varphi(\bar{x})=\overline{\operatorname{conv}}^{*}\left(\partial_{M} \varphi(\bar{x})\right)$, where $\partial_{M}$ stands for Mordukhovich's (limiting) subdifferential, see [7, Theorem $3.57(i i)]$. In that case, one can show that

$$
\partial^{\circ} \varphi(\bar{x}) \subseteq \overline{\operatorname{conv}}^{*}\left(\bigcup_{\bar{y} \in S(\bar{x})}\left\{x^{*} \in X^{*} \mid\left(x^{*}, 0\right) \in \partial^{\circ} f(\bar{x}, \bar{y})+N_{M}(\operatorname{gph} F,(\bar{x}, \bar{y}))\right\}\right),
$$

where $N_{M}(\operatorname{gph} F,(\bar{x}, \bar{y}))$ denotes the limiting normal cone of gph $F$ at $(\bar{x}, \bar{y})$. Moreover, according to [7, Theorem $3.57(i)$ ], we also have

$$
N_{C}(\operatorname{gph} F,(\bar{x}, \bar{y}))=\overline{\operatorname{conv}}^{*}\left(N_{M}(\operatorname{gph} F,(\bar{x}, \bar{y}))\right) .
$$

It then follows that an upper estimate obtained in this way is significantly smaller than the one derived by us in Theorem 3.1. Thus, our results are more relevant in the case in which the Asplund assumption on the spaces is not satisfied.

We conclude the section with an example that illustrates an additional drawback of the upper estimate (3.14) in Theorem 3.1. Specifically, we show that, depending on the representation of $\varphi$ by the set-valued mapping $F$, the estimates obtained can be both trivial and exact.

Example 3.1. Set $X=Y=\mathbb{R}$, and consider $F_{1}, F_{2}: \mathbb{R} \rightrightarrows \mathbb{R}, f: \mathbb{R}^{2} \rightarrow \mathbb{R}$ defined respectively as

$$
\forall(x, y) \in \mathbb{R}^{2}: F_{1}(x)=\{|x|\}, F_{2}(x)=F_{1}(x)+[0,1], f(x, y)=y .
$$

Then, we have $|\cdot|=\varphi(\cdot)=\inf _{y \in F_{1}(\cdot)} f(\cdot, y)=\inf _{y \in F_{2}(\cdot)} f(\cdot, y)$. Let us then analyze the upper estimate (3.14) for each case when $\bar{x}=0$.

(i) Case 1: $F=F_{1}$.

Since $S(0)=\{0\}$ and $\partial^{\circ} f(0,0)=\left\{\left(\begin{array}{l}0 \\ 1\end{array}\right)\right\}$, the upper estimate is exactly

$$
U_{1}:=\overline{\operatorname{conv}}^{*}\left(\bigcup_{\left(x^{*}, y^{*}\right) \in\{(0,1)\}}\left[x^{*}+D_{C}^{*} F_{1}(0,0)\left(y^{*}\right)\right]\right)=D_{C}^{*} F_{1}(0,0)(1) .
$$

Furthermore, it is well known that $N_{C}(\mathrm{gph}|\cdot|,(0,0))=\mathbb{R}^{2}$, so that we actually have $U_{1}=$ $\mathbb{R}$. 
(ii) Case 2: $F=F_{2}$.

Similarly to the previous case, we find that the upper estimate is

$$
U_{2}:=D_{C}^{*} F_{2}(0,0)(1)=[-1,1]=\partial^{\circ} \varphi(0),
$$

and hence the equality holds.

\section{CONCLUSiOnS}

In our paper, we derived an upper estimate of the Clarke subdifferential of marginal functions in Banach spaces. The results are mostly of interest in the case in which the underlying spaces are Banach, but do not necessarily satisfy the Asplund condition. Using our results, it is possible to derive necessary optimality conditions for the solutions of set-valued optimization problems in the setting of general Banach spaces analogously to the procedure in [16] for Asplund spaces.

\section{REFERENCES}

[1] D.T.V. An, N.D. Yen, Differential stability of convex optimization problems under inclusion constraints, Appl. Anal., 94 (2015), 108-128.

[2] V. Bondarevsky, A. Leschov, L. Minchenko, Value functions and their directional derivatives in parametric nonlinear programming, J. Optim. Theory Appl. 171 (2016), 440-464.

[3] B. Luderer, Directional derivative estimates for the optimal value function of a quasidifferentiable programming problem, Math. Program. 51 (1991), 333-348.

[4] L.I. Minchenko, Directional differentiation of marginal functions in mathematical programming problems, Kibernet. Sistem. Anal. 189 (1991), 70-77.

[5] R.I. Bots, An upper estimate for the Clarke subdifferential of an infimal value function proved via the Mordukhovich subdifferential, Nonlinear Anal. 75 (2012), 1141-1146.

[6] J.-B. Hiriart-Urruty, Gradients généralisés de fonctions marginales, SIAM J. Control Optim. 16 (1978), 301-316.

[7] B. Mordukhovich, Variational Analysis and Generalized Differentiation. I, vol. 330 of Grundlehren der Mathematischen Wissenschaften [Fundamental Principles of Mathematical Sciences], Springer-Verlag, Berlin, 2006.

[8] B.S. Mordukhovich, N.M. Nam, N.D. Yen, Subgradients of marginal functions in parametric mathematical programming, Math. Program. 116 (2009), 369-396.

[9] L. Thibault, On subdifferentials of optimal value functions, SIAM J. Control Optim. 29 (1991), 1019-1036.

[10] F.H. Clarke, Optimization and Nonsmooth Analysis, vol. 5 of Classics in Applied Mathematics, Society for Industrial and Applied Mathematics (SIAM), second ed., Philadelphia, 1990.

[11] I. Ekeland, On the variational principle, J. Math. Anal. Appl. 47 (1974), 324-353.

[12] I. Ekeland, Nonconvex minimization problems, Bull. Amer. Math. Soc. 1 (1979), 443-474.

[13] W. Schirotzek, Nonsmooth Analysis, Universitext, Springer, Berlin, 2007.

[14] J. Jahn, Introduction to the Theory of Nonlinear Optimization, third ed., Springer, Berlin, 2007.

[15] A. Göpfert, H. Riahi, C. Tammer, C. Zalinescu, Variational Methods in Partially Ordered Spaces, Springer, New York, 2003.

[16] G. Bouza, E. Quintana, C. Tammer, V. Tuan, The Fermat rule for set optimization problems with Lipschitzian set-valued mappings, J. Nonlinear Convex Anal. 21 (2020), 1137-1174. 